\title{
Impact of Breed on the Fecal Microbiome of Dogs under the Same Dietary Condition
}

\author{
Kondreddy Eswar Reddy ${ }^{1}$, Hye-Ran Kim ${ }^{1}$, Jin Young Jeong ${ }^{1}$, Kyoung-Min So ${ }^{2}$, Seul Lee ${ }^{1}$, Sang Yun Ji ${ }^{1}$, \\ Minji Kim ${ }^{1}$, Hyun-Jung Lee ${ }^{3}$, Sungdae Lee ${ }^{1}$, Ki-Hyun Kim ${ }^{1}$, and Minseok Kim ${ }^{4 *}$ \\ ${ }^{1}$ Animal Nutrition and Physiology Team, National Institute of Animal Science, Wanju 55365, Republic of Korea \\ ${ }^{2}$ Planning and Coordination Division, National Institute of Animal Science, Wanju 55365, Republic of Korea \\ ${ }^{3}$ Dairy Science Division, National Institute of Animal Science, Cheonan 31000, Republic of Korea \\ ${ }^{4}$ Department of Animal Science, College of Agriculture and Life Sciences, Chonnam National University, Gwangju 61186, Republic of Korea
}

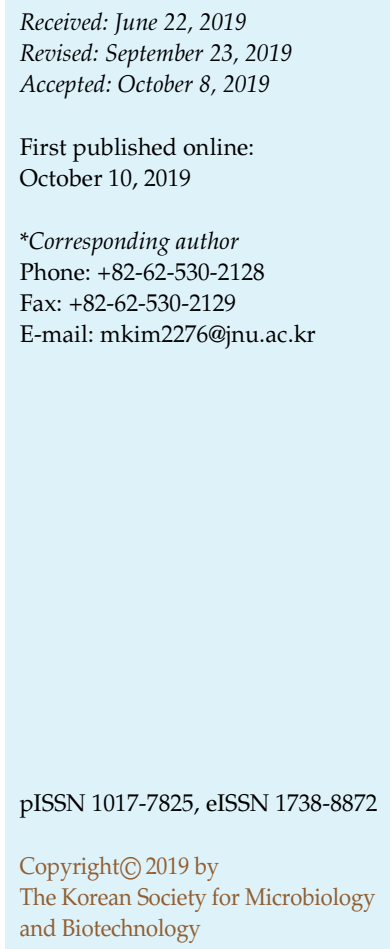

The gut microbiome influences the health and well-being of dogs. However, little is known about the impact of breed on the fecal microbiome composition in dogs. Therefore, we aimed to investigate the differences in the fecal microbiome in three breeds of dog fed and housed under the same conditions, namely eight Maltese ( $8.0 \pm 0.1$ years), eight Miniature Schnauzer $(8.0 \pm 0.0$ years $)$, and nine Poodle dogs $(8.0 \pm 0.0$ years $)$. Fresh fecal samples were collected from the dogs and used to extract metagenomic DNA. The composition of the fecal microbiome was evaluated by $16 \mathrm{~S}$ rRNA gene amplicon sequencing on the MiSeq platform. A total of 840,501 sequences were obtained from the 25 fecal samples and classified as Firmicutes (32.3-97.3\% of the total sequences), Bacteroidetes (0.1-62.6\%), Actinobacteria (0.2-14.7\%), Fusobacteria (0.0-5.7\%), and Proteobacteria (0.0-5.1\%). The relative abundance of Firmicutes was significantly lower in the Maltese dog breed than that in the other two breeds, while that of Fusobacteria was significantly higher in the Maltese than in the Miniature Schnauzer breed. At the genus level, the relative abundance of Streptococcus, Fusobacterium, Turicibacter, Succinivibrio, and Anaerobiospirillum differed significantly among the three dog breeds. These genera had no correlation with age, diet, sex, body weight, vaccination history, or parasite protection history. Within a breed, some of these genera had a correlation with at least one blood chemistry value. This study indicates that the composition of the fecal microbiome in dogs is affected by breed.

Keywords: Dog, fecal microbiome, breed, dietary condition, bacterial abundance

\section{Introduction}

The canine digestive tract is colonized by millions of bacteria that exert a positive and dynamic effect on host health [1]. These bacteria are thought to greatly colonize the colon, and play an important role in numerous functions in the host. According to Honneffer et al. [2], the expected gut microbiota of animals and humans comprises $10^{10}-10^{14}$ microbial cells; this number may be similar to or 10 times greater than the number of host cells. A number of studies show microbial composition and the relationship of specific microbes with the persistence of clinical health signs and during a variety of disease states and conditions such as gastrointestinal inflammation, obesity, and diarrhea $[3,4]$.

Owing to the easy sample collection method, fecal samples are generally used to define the intestinal bacteria, hence the term fecal microbiota. However, this reflects microbial groups present in the distal part of the colon more than in the proximal parts of the intestine [5, 6]. Studies using canine models have emphasized the importance of the intestinal microbiota in harvesting energy from the diet. It has already been identified that the fecal microbiome is affected by numerous aspects. Earlier studies exposed that gut health status is one of the most robust factors influencing 
the composition and diversity of the fecal microbiome [3].

The effect of host health and physiology on microbial variations was also examined in dogs [4]. Gastrointestinal (GI) diseases are related to changes in the intestinal microbiota. According to Suchodolski et al. [7], dogs which suffered from diarrhea showed significant differences in fecal microbial communities compared to healthy dogs, and the fecal dysbiosis in acute diarrhea might be related to changes in systemic metabolic states. Other GI diseases such as inflammatory bowel diseases (IBD) can be linked to modifications in the microbiota of the small intestine and feces [2]. Dogs with calcium oxalate stones also have varied diversity in intestinal microbiota when compared with healthy dogs [8].

When compared with humans and domestic livestock, dogs have a comparatively simple gastrointestinal tract (GIT) and do not depend on microbiota for energy. However, current studies have exposed that a dog's GIT microbial community is composed of some hundred microbial phylotypes [9]. The primary purpose of studying canine microbiota is to obtain a better understanding of the taxonomy, dynamics, and functions of GI microbial communities, as well as the interactions between microbial members, the substances created and consumed by microbes, the influence of diet, drugs, and housing environmental factors, relations with the host, and the variances between healthy and diseased populations [10].

The intestinal microbiota in dogs, however, are yet to be scientifically characterized at the species level, and it is difficult to relate knowledge based on bacteria culture methods [11] to the newer high-throughput cultureindependent data. Furthermore, the bottlenecks associated with the high-throughput sequencing techniques being developed are obviously due to computational capacity and biological clarifications by investigators. Over the past few years, it is clear that studying the whole microbiome is necessary to understand its role in the host rather than studying phylogeny alone. Therefore, analyses using shotgun sequencing of all DNA in a sample provides information on the functional ability of the microbial genome. Little information is available on the relationships among intestinal microbiota and the feed, age, and sex of the canine species. To the best of our knowledge, this is the first study to evaluate the role of GI microbiota in different canine breeds. Thus, the aims of the present study were to examine the composition of the intestinal microbiota in three different dog breeds fed and housed under the same conditions, to clarify the breed-dependent transitions, and compare the composition of potentially beneficial bacteria.

\section{Materials and Methods}

\section{Ethical Statement}

This study was approved by the Institutional Animal Care and Use Committee of the National Institute of Animal Science, South Korea (No. 2017-256, 4 July 2017).

\section{Study Animals and Diet}

The experiment was performed using three breeds of clinically healthy female canines, namely eight Maltese $(8.0 \pm 0.1$ years), eight Miniature Schnauzer ( $8.0 \pm 0.0$ years), and nine Poodle dogs ( $8.0 \pm 0.0$ years). All dogs were housed indoors in separate blocks, and given free access to water. Detailed information of canine breed name, age, sex, body weight, vaccination history, parasite protection history, and diet information is provided in Table 1. The dogs did not show any signs of disease and were generally in good condition. None of the dogs showed any signs of GI diseases or received antibiotics in the two months before fecal sample collection. All three canine breeds were fed daily with the same basal canine diet 5LL9 (LabDiet, USA). The diet comprised 27\% crude protein, $10 \%$ crude fat, $5 \%$ crude fiber and included the following mineral nutrients: $7.7 \%$ ash, $1.8 \% \mathrm{Ca}, 0.95 \% \mathrm{P}, 0.7 \% \mathrm{~K}$, $0.21 \% \mathrm{~S}, 0.16 \% \mathrm{Mg}, 0.66 \% \mathrm{Cl}, 0.41 \% \mathrm{Na}, 13.2 \mathrm{ppm} \mathrm{F}, 387 \mathrm{ppm} \mathrm{Fe}$, 130 ppm Zn, 73 ppm Mn, 14 ppm Cu, 2.13 ppm I, 0.92 ppm Co, 1.18 ppm $\mathrm{Cr}$, and 0.43 ppm Se.

\section{Blood Sample Collection and Analysis}

Blood was collected from all the breeds via jugular venipuncture

Table 1. Breed type, age, gender, weight, vaccination and diets information of dogs.

\begin{tabular}{|c|c|c|c|c|c|c|}
\hline Breed type ${ }^{1}$ & $\begin{array}{c}\text { Age } \\
\text { (year) }\end{array}$ & Gender & $\begin{array}{l}\text { Average weight } \\
(\mathrm{kg})\end{array}$ & $\begin{array}{c}\text { Vaccination } \\
\text { for each } \mathrm{DOG}^{2}\end{array}$ & $\begin{array}{l}\text { Parasite protection } \\
\text { for each } \mathrm{DOG}^{3}\end{array}$ & Diet history $^{4}$ \\
\hline Poodle & 8 & Female & 4.60 & Yearly (DHLPP vaccine) & Monthly (Advocate) & LabDiet 5LL9 \\
\hline Maltese & 8 & Female & 3.78 & Yearly (DHLPP vaccine) & Monthly (Advocate) & LabDiet 5LL9 \\
\hline Miniature Schnauzer & 8 & Female & 7.64 & Yearly (DHLPP vaccine) & Monthly (Advocate) & LabDiet 5LL9 \\
\hline
\end{tabular}

${ }^{1}$ Poodle $n=9$; Maltese $n=8$; Miniature Schnauzer $n=8$

${ }^{2}$ DHLPP vaccination protects the dogs from distemper, hepatitis, Leptospirosis, parainfluenza, and parvovirus diseases.

${ }^{3}$ Advocate injections protects from various parasites such as mites and worms.

${ }^{4}$ All three canine breeds were fed daily with the same Lab Diet 5LL9 commercial diet. 
into an evacuated red top tube without any anticoagulant for biochemistry panel analysis. Blood biochemistry was analyzed under standard conditions on a clinical chemistry analyzer (ProCyte Dx Hematology Analyzer; IDEXX Laboratories, Inc., USA). Blood biochemistry parameters evaluated included albumin, alkaline phosphatase (ALP), alanine aminotransferase (ALT), aspartate aminotransferase (AST), total bilirubin, blood urea nitrogen (BUN), calcium, cholesterol, creatinine, glucose, perfusion index $(\mathrm{Pi})$, gamma-glutamyl transferase (GGT), and total protein.

\section{Sampling and Processing}

The fecal microbiota composition was examined in all three canine breeds (or 25 dogs). After feeding the animals for three weeks, fresh individual fecal samples were collected aseptically into polythene zip bags and immediately transferred to the laboratory.

\section{DNA Extraction and 16 rRNA Gene Sequencing}

Total community DNA was extracted from the 25 fecal samples using the $\mathrm{RBB}+\mathrm{C}$ bead-beating method [12], and stored at $-20^{\circ} \mathrm{C}$ before sequencing. From each DNA sample, a library was generated using the 341F (5'-CCTACGGGNGGCWGCAG-3') and 805R (5'-GACTACHVGGGTATCTAATCC-3') primers to amplify $16 \mathrm{~S}$ rRNA gene amplicons, which produced approximately $450 \mathrm{bp}$ products for targeting the V3-V4 region, sequenced on the MiSeq platform (Illumina, USA). Paired reads were collected using the FLASH program [13]. The assembled fecal microbiome sequences were demultiplexed and microbiome analyses were performed using QIIME v.1.9.1 software [14]. Finally, the subsequent highquality sequences were classified into taxa using BLASTN v.2.4.0 against the National Center for Biotechnology Information (NCBI)
Reference Sequence (RefSeq) database (http://www. ncbi. nlm. nih. gov/RefSeq/). The alpha diversity was analyzed through number of OTUs, Chao1, Shannon diversity index, and Simpson diversity index, while the beta diversity was analyzed through the unweighted principal coordinate analysis (PCoA) plot. Microbial enterotypes were analyzed using Dirichlet multinomial mixture models [15] with Mothur v.1.42.3 software [16].

\section{Statistical Analysis}

Taxa demonstrating on average $\geq 0.2 \%$ of the total sequences were considered major taxa, and used for statistical analysis. The taxonomic composition of fecal bacteria was compared among the three canine groups based on the means of the relative taxonomic abundances (i.e., reads of a taxon/total reads in a sample), as mentioned in our previous study [17]. The mean proportion of each taxon, blood chemistry value, and diversity index was compared among the three canine breeds using analysis of variance (ANOVA), followed by Duncan's test using XLSTAT statistical software (Addinsoft, USA). A $p$ value of $\leq 0.05$ was considered statistically significant. Correlations between bacterial genera and other factors such as body weight and health conditions were analyzed by Spearman's correlation analysis using XLSTAT statistical software (Addinsoft).

\section{Results}

\section{Blood Biochemistry Results in Different Breeds}

Table 2 shows the median range of whole-blood parameters for three different dog breeds. Albumin, glucose, and triglycerides were significantly different, and the remaining

Table 2. Concentrations of selected blood Biochemistry in the clinically healthy Poodle, Maltese, and Miniature Schnauzer breeds.

\begin{tabular}{|c|c|c|c|c|c|}
\hline Analyte $^{1}$ & Normal range $^{2}$ & Poodle & Maltese & Miniature Schnauzer & $p$-value \\
\hline Albumin & $2.5-4.0 \mathrm{~g} / 1$ & $2.92 \pm 0.06^{\mathrm{a}}$ & $2.69 \pm 0.87^{b}$ & $3.04 \pm 0.06^{\mathrm{a}}$ & 0.04 \\
\hline ALP & $10-150 \mathrm{u} / 1$ & $92.60 \pm 12.81$ & $83.64 \pm 16.37$ & $135.17 \pm 27.83$ & 0.30 \\
\hline AST (GOT) & $5-55 \mathrm{u} / 1$ & $41.04 \pm 2.03$ & $37.48 \pm 2.18$ & $66.08 \pm 12.81$ & 0.08 \\
\hline Total Bilurubin & $0.1-0.3 \mathrm{mg} / \mathrm{dL}$ & $0.14 \pm 0.01$ & $0.14 \pm 0.01$ & $0.15 \pm 0.01$ & 0.58 \\
\hline BUN & $6-25 \mathrm{mg} / \mathrm{dL}$ & $15.15 \pm 0.35$ & $15.69 \pm 0.49$ & $16.36 \pm 0.94$ & 0.59 \\
\hline Calcium & $8.9-11.4 \mathrm{mg} / \mathrm{dL}$ & $9.50 \pm 0.23$ & $9.36 \pm 0.12$ & $10.21 \pm 0.24$ & 0.06 \\
\hline Cholesterol & $92-324 \mathrm{mg} / \mathrm{dL}$ & $168.93 \pm 11.41$ & $161.84 \pm 9.37$ & $170.23 \pm 11.50$ & 0.82 \\
\hline Creatinine & $0.5-1.6 \mathrm{mg} / \mathrm{dL}$ & $0.72 \pm 0.02$ & $0.63 \pm 0.02$ & $0.77 \pm 0.04$ & 0.18 \\
\hline Glucose & 70-150 mg/dL & $80.24 \pm 2.65^{\mathrm{b}}$ & $94.17 \pm 2.49^{a}$ & $78.60 \pm 3.89^{b}$ & 0.01 \\
\hline $\mathrm{Pi}$ & $0.14-9.7 \mathrm{mg} / \mathrm{dL}$ & $4.84 \pm 0.25$ & $4.91 \pm 0.20$ & $4.25 \pm 0.11$ & 0.18 \\
\hline GGT & $0-14 \mu / 1$ & $7.57 \pm 0.42$ & $7.06 \pm 0.34$ & $6.66 \pm 0.48$ & 0.33 \\
\hline
\end{tabular}

Data are presented as mean $( \pm$ SEM).

${ }^{1}$ ALP, Alkaline Phosphatase; ALT, Alanine Aminnotransferase; AST, Aspertate transaminase; BUN, Blood Urea Nitrogen; Pi, Perfusion Index; GGT, Gamma glutamyl transferase; HDL, High density lipoprotein; LDL, Low density lipoprotein.

${ }^{2}$ Normal ranges are from published reports.

${ }^{a, b}$ Values with different letters within the same row are different $(p<0.05)$. 
parameters did not show any significant differences between Poodle, Maltese, and Miniature Schnauzer breeds under the same dietary conditions. The characteristics of the blood biochemistry panel of the three breeds were determined to be within normal ranges.

\section{The Composition of Intestinal Microbiota in Different Breeds}

A total of 840,501 cleaned ribosomal rRNA sequences with a 500-nucleotide average read length were obtained from the total fecal sample (including all three breeds). Of these, 288,608 were obtained from the Poodle group (average sequence number 32,067; range 28,019-39,548), 264,559 from the Maltese group (average sequence number 33,069; range 28,950-42,403), and 287,334 from the Miniature Schnauzer group (average sequence number 35,916; range 28,798-45,278). Of the total 840,501 sequences, Poodle, Maltese, and Miniature Schnauzer dogs shared 34.34\%, $31.48 \%$ and $34.19 \%$ sequences, respectively.

As shown in Table 3 and Fig. 1A, Firmicutes and Bacteroidetes were the most abundant phyla and occupied more than $90 \%$ of the total sequences in all three canine breeds. Firmicutes was the most abundant phylum in Miniature Schnauzer, Poodle, and Maltese breeds, occupying $80.17 \%, 78.26 \%$, and $57.64 \%$ of the total sequences, respectively (Fig. 1B). The second most dominant phylum was Bacteroidetes with abundances of $33.41 \%, 15.79 \%$, and $14.88 \%$ in Maltese, Poodle, and Miniature Schnauzer breeds, respectively (Fig. 1C). The third greatest abundant phylum is Proteobacteria (Fig. 1D). The subsequent most abundant phyla were Actinobacteria, and Fusobacteria of all cleaned sequences. Actinobacteria showed greater abundances in the Maltese (4.56\%) and Poodle $(3.32 \%)$ breeds than that in the Miniature Schnauzer breed (1.98\%). Protobacteria and Fusobacteria abundances were 1.75\% (Maltese); 1.48\% (Miniature Schnauzer); and 1.07\% (Poodle) and $2.63 \%$ (Maltese); $1.55 \%$ (Poodle); and 1.49\% (Miniature Schnauzer), respectively. In addition, the gut microbial taxonomic compositions at the phylum and family level from each dog of the three canine breeds are shown in Fig. 2.

\section{Taxonomic Composition of Fecal Bacteria}

Among all phylum groups, Firmicutes was the most abundant phylum $(p<0.05)$ in the three fecal sample groups (Table 3, Fig. 1B). The abundance of Firmicutes varied among the canine groups, and comprised 11 genera, indicating at least $\geq 0.2 \%$ of the sequences in the total reads. Among these genera, only Streptococcus and Turicibacter showed significant differences $(p \leq 0.05)$ among the three breeds. Streptococcus was the most abundant in Miniature Schnauzer (17.83\%), moderate in Poodle $(9.38 \%)$, and the least abundant in Maltese (3.83\%) fecal samples. The abundance of Turicibacter was significantly greater $(p \leq$ $0.05)$ in Maltese $(2.60 \%)$ and Poodle $(2.46 \%)$ fecal samples than that in Miniature Schnauzer (0.38\%) samples. Among the 11 genera in the phylum Firmicutes, Lactobacillus was the most dominant in all three breeds, but there were no significant differences among them. In particular, Lactobacillus showed the greatest abundance in Poodle $(34.14 \%)$ fecal samples than that in Miniature Schnauzer $(18.75 \%)$ and Maltese (17.50\%) samples. Residual genera in Firmicutes, such as Megamonas, Streptococcus, Blautia, and Catenibacterium also showed high abundances but there were no significant differences among the breeds. Megamonas showed high abundances in the Miniature Schnauzer $(17.81 \%)$ breed, but there was little difference between

Table 3. The relative abundance of major taxa in three canine breeds.

\begin{tabular}{|c|c|c|c|c|c|c|c|}
\hline \multirow[b]{2}{*}{ Classification } & \multirow{2}{*}{$\begin{array}{l}\text { Collective data } \\
\text { average }^{1}\end{array}$} & \multicolumn{5}{|c|}{ Percentage of total sequences in different breed ${ }^{2}$} & \multirow{2}{*}{$\begin{array}{l}\text { No. of dogs with } \\
\text { detectable taxon }\end{array}$} \\
\hline & & $\begin{array}{l}\text { Poodle dogs } \\
\quad(n=8)\end{array}$ & $\begin{array}{l}\text { Maltese dogs } \\
\quad(n=8)\end{array}$ & $\begin{array}{l}\text { Miniature Schnauzer dogs } \\
\qquad(n=9)\end{array}$ & SEM & $p$-value & \\
\hline Firmicutes & 72.02 & $78.26^{\mathrm{a}}$ & $57.64^{\mathrm{b}}$ & $80.17^{\mathrm{a}}$ & 0.039 & 0.009 & 25 \\
\hline Streptococcus & 10.35 & $9.38^{\mathrm{ab}}$ & $3.83^{\mathrm{b}}$ & $17.83^{\mathrm{a}}$ & 0.486 & 0.042 & 24 \\
\hline Turicibacter & 1.81 & $2.46^{\mathrm{ab}}$ & $2.60^{\mathrm{a}}$ & $0.38^{\mathrm{b}}$ & 0.305 & 0.009 & 24 \\
\hline Proteobacteria & 1.43 & 1.07 & 1.75 & 1.48 & 0.211 & 0.478 & 25 \\
\hline Succinivibrio & 0.20 & $0.01^{\mathrm{b}}$ & $0.02^{\mathrm{b}}$ & $0.57^{\mathrm{a}}$ & 0.321 & 0.003 & 15 \\
\hline Anaerobiospirillum & 0.19 & $0.37^{\mathrm{a}}$ & $0.17^{\mathrm{ab}}$ & $0.02^{\mathrm{b}}$ & 0.356 & 0.003 & 17 \\
\hline Fusobacteria & 1.89 & $1.55^{\mathrm{ab}}$ & $2.63^{\mathrm{a}}$ & $1.49^{\mathrm{b}}$ & 0.170 & 0.050 & 25 \\
\hline Fusobacterium & 1.90 & $1.55^{\mathrm{ab}}$ & $2.63^{\mathrm{a}}$ & $1.53^{\mathrm{b}}$ & 0.170 & 0.050 & 25 \\
\hline
\end{tabular}

${ }^{1}$ Sequences obtained from all 25 fecal samples

${ }^{2}$ Values represent means.

${ }^{a, b}$ Within a row, means with a different subscript were different $(p<0.05)$. 

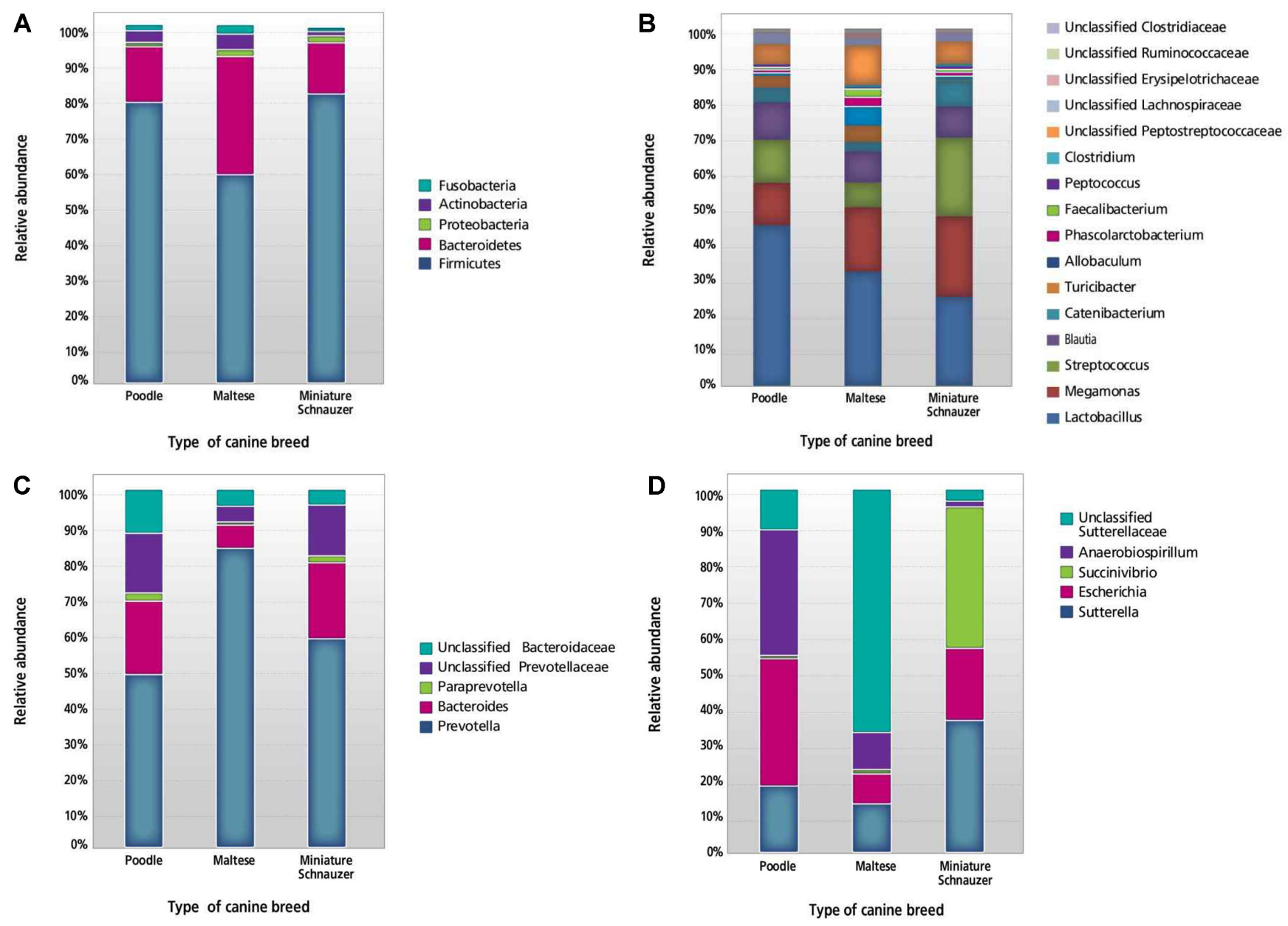

Fig. 1. Stacked histograms showing, the relative abundances of bacterial diversity for the phylum, family and genus-level in Poodle, Maltese and Miniature Schnauzer breeds. Breed name shown on the $\mathrm{X}$ axis and percentage reads classified on the $\mathrm{Y}$ axis.

(A) Showed the relative abundances of taxa within all phyla, (B) showed the abundance of Firmicutes, (C) shown abundance of Bacteroidetes, and (D) shown the abundance of Proteobacteria.

Maltese (10.51\%) and Poodle (9.63\%) breeds. Blautia showed rich abundance in the Poodle (8.33\%) and Miniature Schnauzer (7.12\%) breeds compared to the Maltese (5.17\%) breed. There were non-significant differences in abundance $(p>0.05)$ in the following genera among the three canine fecal samples: Turicibacter (1.81\%), Allobaculum (1.63\%), Phascolarctobacterium (0.94\%), Faecalibacterium (0.83\%), Peptococcus $(0.46 \%)$, and Clostridium $(0.28 \%)$. However, some of the Firmicutes sequences could not be classified into a recognized genus in all three breeds; here, the abundances of five unclassified groups $(p>0.05)$ in the collective data were Peptostreptococcaceae (5.42\%), Lachnospiraceae $(1.60 \%)$, Erysipelotrichaceae (0.67\%), Ruminococcaceae $(0.34 \%)$, and Clostridiaceae $(0.17 \%)$.

Bacteroidetes was the second most dominant phylum in the three breeds $(21.36 \%)$, and there were three genera and two unclassified families in this phylum (Fig. 1C), but there were no significant differences $(p>0.05)$ among the three breeds. Within Bacteroidetes, Prevotella was the most abundant genera $(p>0.05)$ and showed the greatest abundance in the Maltese breed (26.79\%) when compared with that in the Miniature Schnauzer $(8.96 \%)$ and Poodle breeds $(7.42 \%)$. For the remaining known genera in the collective data, Bacteroides and Paraprevotella showed abundances of $2.83 \%$ and $0.22 \%$, respectively. Within Bacteroidetes, unclassified Prevotellaceae (2.12\%) and unclassified Bacteroidaceae (1.26\%) did not show significant differences $(p>0.05)$ among the three breed samples.

Within the phylum Proteobacteria, Sutterella, Escherichia, Succinivibrio, and Anaerobiospirillum genera, and one unclassified Sutterellaceae family were identified (Fig. 1D). Within these genera, Succinivibrio showed significantly $(p<$ 

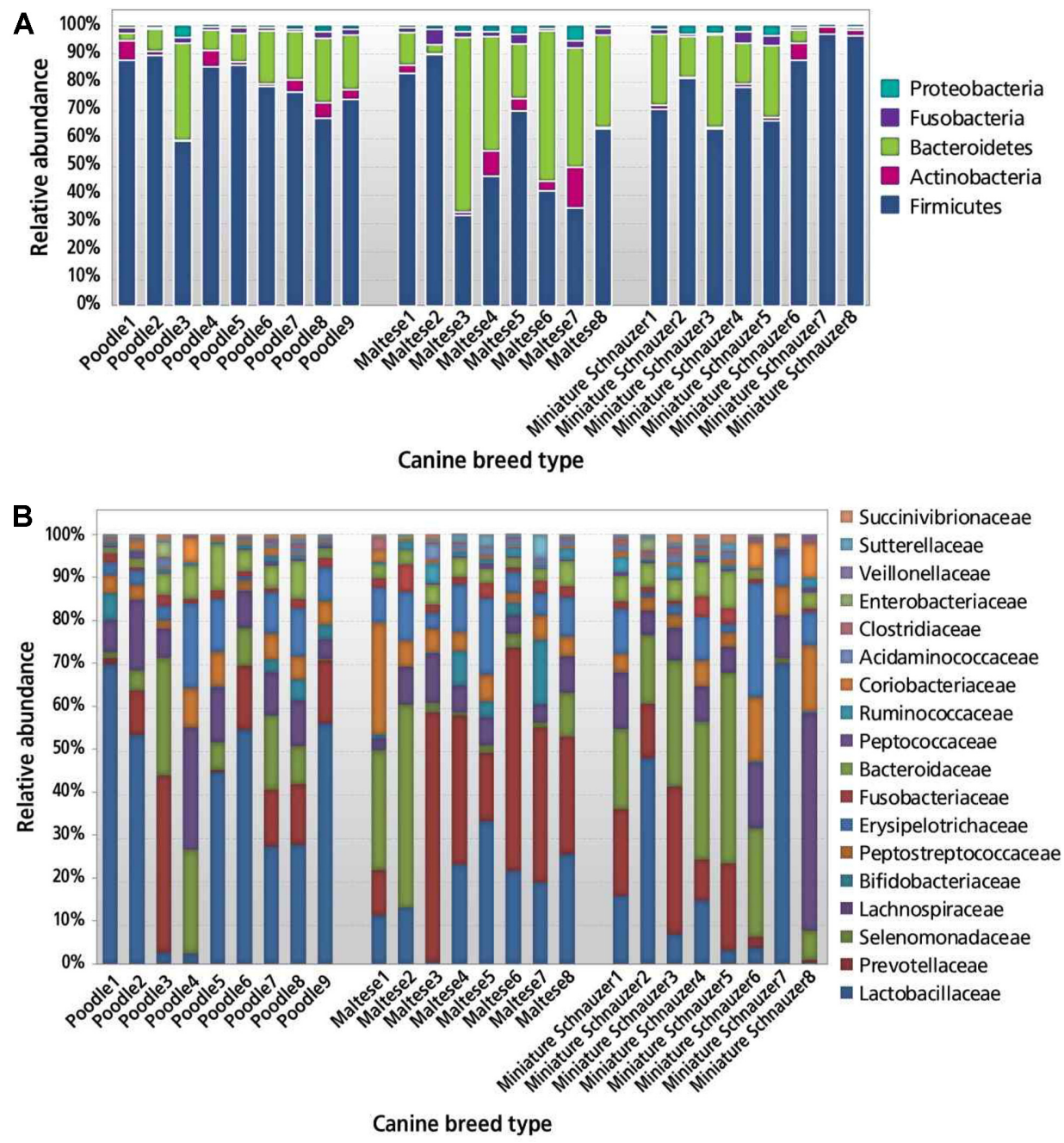

Fig. 2. Microbial taxonomic profiles from each dog of three canine breeds at the phylum (A), and family (B) levels, classified by the representation of $>1 \%$ of the total sequences.

Taxonomic compositions of each dog among the Poodle, Maltese and Miniature Schnauzer breeds were compared based on the relative abundance.

$0.05)$ high abundance $(0.57 \%)$ in the Miniature Schnauzer breed, while Poodle $(0.01 \%)$ and Maltese $(0.02 \%)$ breeds showed low abundances (Table 3). Anaerobiospirillum showed significantly high abundance $(p<0.05)$ in the Poodle $(0.37 \%)$, moderate in the Maltese $(0.17 \%)$, and low abundance in the Miniature Schnauzer $(0.2 \%)$ breed. Actinobacteria was the third most dominant phylum in three canine breeds $(3.29 \%)$, but within this phylum Bifidobacterium (1.95\%) and Collinsella (1.22\%) genera did not show significant differences $(p>0.05)$ among the breeds. Within the Fusobacteria phylum, the Fusobacterium genus showed significant differences $(p>0.05)$ among the three breeds, where Maltese $(2.63 \%)$ showed a higher abundance than the Poodle $(1.55 \%)$ and Miniature
Schnauzer (1.53\%) breeds (Table 3).

Two canine gut microbiome enterotypes were identified in the three breeds. Six individuals, including five Maltese dogs and one Poodle dog, were categorized as having enterotype 1, characterized by high levels of Prevotella but a lower abundance of Megamonas. Meanwhile, the other 19 individuals, including two Maltese, eight Miniature Schnauzer, and eight Poodle dogs, were categorized as having enterotype 2, which comprises high levels of Megamonas but low levels of Prevotella.

\section{Fecal Microbial Diversity}

Alpha diversity indices (number of OTUs, Chao1, Shannon diversity index, and Simpson diversity index) were not 
different $(p>0.05)$ among the three breeds (Table 4). In addition, beta diversity PCoA plot showed that fecal microbiomes of the three breeds were slightly separated, indicating that the composition of fecal microbiota is different among the three canine breeds (Fig. 3). The composition of fecal microbiota in Maltese dogs was more distantly separated compared to the other two canine breeds.

\section{Correlation Analysis}

Only genera that were significantly affected by breeds were considered in the correlation analysis. No genus had a correlation with body weight in any of the three breeds (Fig. 4). Streptococcus did not have a correlation with blood chemistry values in the three breeds. Fusobacterium was positively correlated with TG $(r=0.762, p=0.017)$ in the Poodle breed but was not correlated with the remaining blood chemistry values in the other two breeds. Positive correlations were found for Turicibacter with ALP $(r=$ $0.798, p=0.010)$ in the Poodle breed, with total bilirubin $(r$ $=0.784, p=0.021)$ in the Maltese breed, and with AST $(r=$ 0.819, $p=0.046)$ in the Miniature Schnauzer breed. Succinivibrio was positively correlated with ALT $(r=0.749$, $p=0.020)$ in the Poodle breed and with glucose $(r=0.815, p$ $=0.048)$ in the Miniature Schnauzer breed.

\section{Discussion}

To date, no studies have compared microbiota within different canine breeds; however, a number of recent studies have investigated the effects of diet on canine gut microbiota using high-throughput DNA sequencing methods, revealing that dietary interferences may affect microbial composition and activity, and with likely effects on canine gut health. We believe that the present study is the first to investigate the effect of canine breed on gut microbiota in dogs fed and housed under the same conditions using Next-generation Sequencing (NGS) technology.

In the current study, we concluded that all three dog breeds were healthy because there were no blood biochemistry panel changes over the baseline value, and no

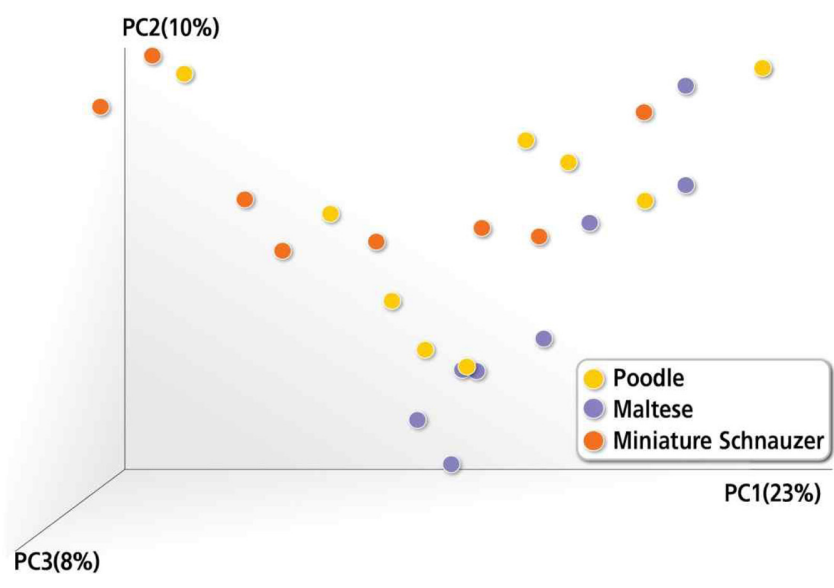

Fig. 3. Unweighted principal coordinate analysis (PCoA) displaying correlations among the three breeds.

The PCoA plot slightly separated fecal microbiome among the three breeds. Red symbols, Miniature Schnauzer; blue symbols, Maltese; yellow symbols, Poodle.

other abnormalities or clinical signs were observed. Since some of the genera that significantly differed among the three breeds were correlated with at least one of the blood chemistry values in one breed but had no correlation with the other two breeds, it will be difficult to conclude that canine fecal microbiota is affected by canine health status. Age was almost the same among dogs in the three breeds, while no genus showed correlations with body weight. Diet, sex, vaccination history, and parasite protection history were the same in all three breeds. Therefore, canine fecal microbiota does not seem to be affected by other factors such as age, body weight, diet, sex, vaccination history, parasite protection history, or health status. In this study, the difference between fecal microbiota seems to be affected by the breed.

The microbial separation by the PCoA plot might have also resulted from the difference in breed because canine fecal microbiota was not affected by other factors except for the breed, as described above. In addition, the PCoA plot shows the fecal microbiota in Maltese dogs to be more distantly separated compared to the other two breeds. This

Table 4. Fecal diversity statistics among three canine breeds.

\begin{tabular}{|c|c|c|c|c|c|}
\hline Canine breed $^{1}$ & No. of observed OTUs & Chao1 & Shannon & Inverse Simpson & Goods coverage \\
\hline Maltage $(n=8)$ & $174.125^{\mathrm{a}}$ & $193.621^{\mathrm{a}}$ & $3.616^{\mathrm{a}}$ & $0.812^{\mathrm{a}}$ & $0.999^{\mathrm{a}}$ \\
\hline Poodle $(n=9)$ & $167.111^{\mathrm{a}}$ & $192.672^{a}$ & $3.709^{\mathrm{a}}$ & $0.828^{\mathrm{a}}$ & $0.999^{\mathrm{a}}$ \\
\hline
\end{tabular}

${ }^{1}$ Means among the three breeds were compared using ANOVA, followed by Duncan's test.

${ }^{a}$ Means with the same superscript letter within a column do not show a significant difference $(p>0.05)$. 
A

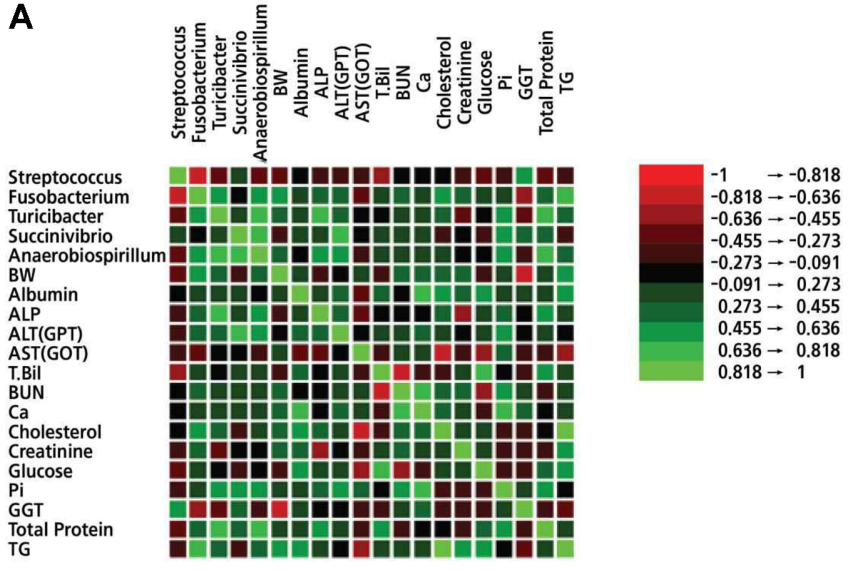

B

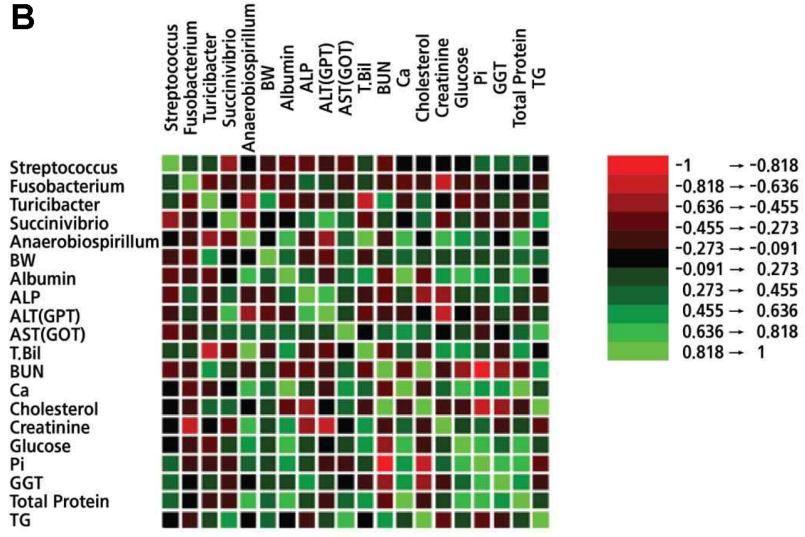

C

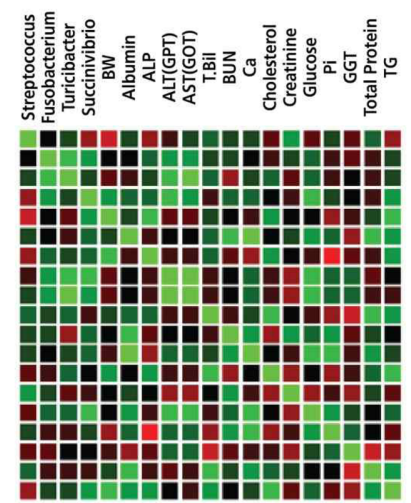

Fig. 4. Correlations between genera affected by breeds and blood chemistry values.

Only genera that are significantly different among the three breeds were considered in correlations. (A) Correlations between genera and blood chemistry values in poodle dogs. (B) Correlations between genera and blood chemistry values in maltese dogs. (C) Correlations between genera and blood chemistry values in miniature schnauzer dogs.

separation might be ascribed to different enterotype classification because most of the Maltese dogs were assigned to enterotype I, while most of the dogs of the other two breeds were assigned to enterotype II.

In the $16 \mathrm{~S}$ rRNA gene analysis on the three dog breeds in our study, the dominant phyla were Firmicutes and Bacteroidetes, which included more than $90 \%$ of the total sequences in all three breeds. Other abundant phyla were Proteobacteria, Actinobacteria, and Fusobacteria. Earlier studies also found that Firmicutes, Bacteroidetes, Proteobacteria, and Fusobacteria were the abundant phyla, even though there were variations in the dogs and the conditions under which they were held, such as age, diet, genetic background, breeding environment, and health [10, $18,19]$.

In the present study, there were significant differences in Firmicutes abundance among the three canine breeds, particularly for Miniature Schnauzer (80.17\%) and Poodle (78.26\%) breeds showing higher abundances than for Maltese dogs (57.64\%). These dissimilarities might be due to differences in breed, individual variations in gut microbiota, or other individual variations in the animals. According to Turnbaugh et al. [20], specifically, gut microbiota is extremely affected by the host genotype and different environmental conditions that are difficult to standardize. Within Firmicutes, there were high abundances of genus Lactobacillus in all three dog breeds, but there were no significant differences among them; although, the Poodle and Miniature Schnauzer breeds had higher abundances than the Maltese dogs. In previous studies, dog fecal samples also had high abundances of Lactobacillus in Poodle [21] and Miniature Schnauzer dogs [22]. According to Swann et al. [23], Lactobacillus plays a key role in bile acid metabolism and affects the absorption of dietary fats and lipid soluble vitamins. Furthermore, Lactobacillus strains have the potential to be used in emerging probiotic food [24]; thus, Lactobacillus identified in the present study might also have the potential to be used as probiotics, especially in Poodle and Miniature Schnauzer dogs.

Streptococcus abundance was also high in all three dog breeds with the greatest percentage in Miniature Schnauzer dogs. Streptococcus plays an important role in the skin and mucosal health of canines. The abundance of these bacteria may suppress the growth of intestinal pathogens by means of competitive exclusion [25, 26], and earlier studies have shown they can improve the immune functions in humans and mice $[27,28]$. The highest abundance of Streptococcus in Miniature Schnauzer dogs explains that gut microbiota is affected by canine breeds. Megamonas and Blautia genera were also abundant in all breeds, but no significant differences were observed among the breeds. However, we 
propose that these bacteria may act as probiotics and improve intestinal health by relocating intestinal pathogens, thereby, increasing immune responses. According to Suchodolski et al. [7], Blautia is common in healthy dogs, but decreases with canine diarrhea, and may play a role in creating short-chain fatty acids.

Within Bacteroidetes, Prevotella was the most abundant genus with no significant difference among the three breeds. Although David et al. [29] showed that the type of feed plays a major role in the abundance of Prevotella, there are no studies on the relationship between canine breed and Prevotella abundance, and this requires further investigation. Observations from earlier studies indicated that Prevotella plays a key role in the digestive process [30]. It seems that Prevotella is core genus that is commonly found irrespective of different breeds.

In the phylum Proteobacteria, Succinivibrio was significantly higher in abundance in Miniature Schnauzer dogs than that in the Poodle and Maltese breeds. According to Petri et al. [31], Succinivibrio showed high abundance, were important bacteria in the gut microbiome of high starch fed cattle, and played a key role in propionate production through the production of upstream succinate [32]. However, there are no studies on Succinivibrio in dog fecal samples; therefore, little is known about the functions of Succinivibrio. The abundance of Anaerobiospirillum also differed significantly among the three dog breeds. Anaerobiospirillum showed higher abundances in Poodle $(0.37 \%)$ and Maltese $(0.17 \%)$ than that in the Miniature Schnauzer $(0.2 \%)$ breed. Misawa et al. [33] found Anaerobiospirillum in a puppy with hemorrhagic diarrhea, but as extensive studies have not been conducted, the importance or pathogenic mechanisms of Anaerobiospirillum in the intestine remain unknown. There were also significant differences in Fusobacterium among the different canine breeds, with the Maltese breed showing higher abundances of Fusobacterium than that in the Poodle and Miniature Schnauzer breeds. Fusobacterium ferment carbohydrates and certain amino acids to produce butyrate, acetate, and other volatile fatty acids [34]. According to Middelbos [35], Fusobacteria was the codominant phylum in healthy canine feces. Thus, we propose that the significant variations found in Fusobacterium among the three breeds in our study may be mucosal differences associated with different canine breeds.

Knowledge on fecal microbiome composition in different dog breeds is important for understanding the relationships between the host and the fecal microbiome composition. This study shows that fecal microbiome composition differs among different dog breeds fed and housed under the same conditions, indicating that host genetic factors may affect fecal microbiome composition in dogs. Understanding these differences may contribute to the development of different feeding strategies for maintaining a healthy gut microbiome composition in different dog breeds.

\section{Acknowledgments}

This work was carried out with the support of the Cooperative Research Program for Agriculture Science and Technology Development (Project No. PJ01283404) and the 2019 RDA Fellowship Program of the National Institute of Animal Sciences, Rural Development Administration, Korea.

\section{Conflict of Interest}

The authors have no financial conflicts of interest to declare.

\section{References}

1. Suchodolski JS. 2011. Intestinal microbiota of dogs and cats: a bigger world than we thought. Veterinary Clinics North America Small Animal Practices.

2. Honneffer JB, Minamoto Y, Suchodolski JS. 2014. Microbiota alterations in acute and chronic gastrointestinal inflammation of cats and dogs. World J. Gastroenterol. 20: 16489-16497.

3. Guard BC, Barr JW, Reddivari L, Klemashevich C, Jayaraman A, Steiner JM, et al. 2015. Characterization of microbial dysbiosis and metabolomics changes in dogs with acute diarrhea. PLoS One 10: e0127259.

4. Handl S, Dowd SE, Garcia-Mazcorro JF, Steiner JM, Suchodolski JS. 2011. Massive parallel 16S rRNA gene pyrosequencing reveals highly diverse fecal bacterial and fungal communities in healthy dogs and cats. FEMS Microbiol. Ecol. 76: 301-310.

5. Eckburg PB, Bik EM, Bernstein CN, Purdom E, Dethlefsen L, Sargent $\mathrm{M}$, et al. 2005. Diversity of the human intestinal microbial flora. Science 308: 1635-1638.

6. Suchodolski JS, Camacho J, Steiner JM. 2008. Analysis of bacterial diversity in the canine duodenum, jejunum, ileum, and colon by comparative $16 \mathrm{~S}$ rRNA gene analysis. FEMS Microbiol. Ecol. 66: 567-578.

7. Suchodolski JS, Markel ME, Garcia-Mazcorro JF, Unterer S, Heilmann RM, Dowd SE, et al. 2012. The fecal microbiome in dogs with acute diarrhea and idiopathic inflammatory bowel disease. PLoS One 7: e51907.

8. Gnanandarajah JS, Johnson TJ, Kim HB, Abrahante JE, Lulich JP, Murtaugh MP. 2012. Comparative faecal microbiota of dogs with and without calcium oxalate stones. J. Appl. Microbiol. 113: 745-756. 
9. Swanson KS, Dowd SE, Suchodolski JS, Middelbos IS, Vester BM, Barry KA, et al. 2011. Phylogenetic and genecentric metagenomics of the canine intestinal microbiome reveals similarities with humans and mice. ISME J. 5: 639-649.

10. Deng P, Swanson KS. 2015. Gut microbiota of humans, dogs and cats: current knowledge and future opportunities and challenges. Br. J. Nutr. 113: S6-S17.

11. Simpson JM, Martineau B, Jones WE, Ballam JM, Mackie RI. 2002. Characterization of fecal bacterial populations in canines: effects of age, breed and dietary fiber. Microbial. Ecol. 44: 186-197.

12. $\mathrm{Yu} \mathrm{Z}$, Morrison M. 2004. Improved extraction of PCR quality community DNA from digesta and fecal samples. Biotechniques 36: 808-812.

13. Magoc M, Salzberg S. 2011. FLASH: Fast length adjustment of short reads to improve genome assemblies. Bioinformatics 27: 2957-2963.

14. Caporaso JG, Kuczynski J, Stombaugh J, Bittinger K, Bushman FD, Costello EK, et al. 2010. QIIME allows analysis of high-throughput community sequencing data. Nat. Methods 7: 335-336.

15. Holmes I, Harris K, Quince C. 2012. Dirichlet multinomial mixtures: generative models for microbial metagenomics. PLoS One 7: e30126.

16. Schloss PD, Westcott SL, Ryabin T, Hall JR, Hartmann M, Hollister EB, et al. 2009. Introducing mothur: open-source, platform-independent, community-supported software for describing and comparing microbial communities. Appl. Environ. Microbiol. 75: 7537-7541.

17. Kim M, Kim J, Kuehn LA, Bono JL, Berry ED, Kalchayanand N, et al. 2014. Investigation of bacterial diversity in the feces of cattle fed different diets. J. Anim. Sci. 92: 683-694.

18. Omatsu T, Omura M, Katayama Y, Kimura T, Okumura M, Okumura A, et al. 2018. Molecular diversity of the faecal microbiota of toy poodles in Japan. J. Vet. Med. Sci. 80: 749754 .

19. Hooda S, Minamoto Y, Suchodolski JS, Swanson KS. 2012. Current state of knowledge: the canine gastrointestinal microbiome. Anim. Health Res. Rev. 13: 78-88.

20. Turnbaugh PJ, Hamady M, Yatsunenko T, Cantarel BL, Duncan A, Ley RE, et al. 2009. A core gut microbiome in obese and lean twins. Nature 457: 480-484.

21. Schmidt M, Unterer S, Suchodolski JS, Honneffer JB, Guard BC, Lidbury JA, et al. 2018. The fecal microbiome and metabolome differs between dogs fed bones and raw food (BARF) diets and dogs fed commercial diets. PLoS One 13(8): e0201279.

22. Hand D, Wallis C, Colyer A, Penn CW. 2013. Pyrosequencing the canine faecal microbiota: breadth and depth of biodiversity. PLoS One 8: e53115.

23. Swann JR, Want EJ, Geier FM, Spagou K, Wilson ID, Sidaway JE, et al. 2011. Systemic gut microbial modulation of bile acid metabolism in host tissue compartments. Proc. Natl. Acad. Sci. USA 108: S4523-S4530.

24. Zielińska D, Rzepkowska A, Radawska A, Zieliński K. 2015. In vitro screening of selected probiotic properties of Lactobacillus strains isolated from traditional fermented cabbage and cucumber. Curr. Microbiol. 70: 183-194.

25. Hudault S, Lievin V, Bernet-Camard MF, Servin AL. 1997. Antagonistic activity exerted in vitro and in vivo by Lactobacillus casei (strain GG) against Salmonella typhimurium C5 infection. Appl. Environ. Microbiol. 63: 513-518.

26. Pascual M, Hugas M, Badiola JI, Monfort JM, Garriga M. 1999. Lactobacillus salivarius CTC2197 prevents Salmonella enteritidis colonization in chickens. Appl. Environ. Microbiol. 65: 4981-4986.

27. Gill HS, Rutherfurd KJ, Prasad J, Gopal PK. 2000. Enhancement of natural and aquired immunity by Lactobacillus rhamnosus (HN001), Lactobacillus acidophilus (HN017) and bifidobacterium lactis (HN019). Br. J. Nutr. 83: 167-176.

28. Vitini E, Alvarez S, Medina M, Medici M, de Budeguer, MV, Perdigon G. 2000. Gut mucosal immunostimulation by lactic acid bacteria. Biocell 24: 223-232.

29. David LA, Maurice CF, Carmody RN, Gootenberg DB, Button JE, Wolfe BE, et al. 2014. Diet rapidly and reproducibly alters the human gut microbiome. Nature 505: 559-563.

30. Fogarty LR, Voytek MA. 2005. Comparison of BacteroidesPrevotella 16S rRNA genetic markers for fecal samples from different animal species. Appl. Environ. Microbiol. 71: 59996007.

31. Petri RM, Schwaiger T, Penner GB, Beauchemin KA, Forster RJ, McKinnon JJ, et al. 2013. Changes in the rumen epimural bacterial diversity of beef cattle as affected by diet and induced ruminal acidosis. Appl. Environ. Microbiol 79: 37443755.

32. Li F, Guan LL. 2017. Metatranscriptomic profiling reveals linkages between the active rumen microbiome and feed efficiency in beef cattle. Apply. Environ. Microbiol. 83: e0061-17.

33. Misawa N, Kawashima K, Kondo F, Kushima E, Kushima K, Vandamme P. 2002. Isolation and characterization of Campylobacter, Helicobacter, and Anaerobiospirillum strains from a puppy with bloody diarrhea. Vet. Microbiol. 87: 353-364.

34. Shah HN, Olsen I, Bernard K, Finegold SM, Gharbia S, Gupta RS. 2009. Approaches to the study of the systematics of anaerobic, gram-negative, nonsporeforming rods: current status and perspectives. Anaerobe 15: 179-194.

35. Middelbos IS, Vester Boler BM, Qu A, White BA, Swanson KS, Fahey Jr GC. 2010. Phylogenetic characterization of fecal microbial communities of dogs fed diets with or without supplemental dietary fiber using 454 pyrosequencing. PLoS One 5: e9768. 